\title{
Article
}

\section{Active Modulation of Surface Plasmon Resonance Wavelengths by Applying an Electric Field to Gold Nanoparticle-Embedded Ferroelectric Films}

K. C. Hsieh, H. L. Chen, D. H. Wan, and J. Shieh

J. Phys. Chem. C, 2008, 112 (31), 11673-11678 • DOI: 10.1021/jp711828k • Publication Date (Web): 15 July 2008

Downloaded from http://pubs.acs.org on December 10, 2008

\section{More About This Article}

Additional resources and features associated with this article are available within the HTML version:

- $\quad$ Supporting Information

- $\quad$ Access to high resolution figures

- $\quad$ Links to articles and content related to this article

- Copyright permission to reproduce figures and/or text from this article

\section{View the Full Text HTML}




\title{
Active Modulation of Surface Plasmon Resonance Wavelengths by Applying an Electric Field to Gold Nanoparticle-Embedded Ferroelectric Films
}

\author{
K. C. Hsieh, ${ }^{\dagger}$ H. L. Chen, ${ }^{*}, \dagger$ D. H. Wan, ${ }^{\dagger}$ and J. Shieh \\ Department of Materials Science and Engineering, National Taiwan University, Taipei, Taiwan, and \\ National Nano Device Laboratory, Hsinchu, Taiwan
}

Received: December 17, 2007; Revised Manuscript Received: May 13, 2008

\begin{abstract}
In this study, we use organic chemical addition and phase-transfer methods to prepare lead zirconate titanate (PZT) composite films embed with large numbers of gold nanoparticles (Au NPs). The surface plasmon resonance (SPR) wavelength in the nanocomposite materials can be modulated cyclically through the application of an external electric field. The Au NPs are embedded homogeneously in the PZT matrix; the PZT crystal size and the metal particle size both increase upon increasing the annealing temperature. The Au/PZT nanocomposite film displays apparent piezoelectric properties in its ferroelectric hysteresis curve that can be used in the active modulation of the SPR wavelengths. The major factor influencing the shift in the SPR absorption in the Au/PZT nanocomposite films is the change in the refractive index of the host PZT matrix.
\end{abstract}

\section{Introduction}

Nanometer-scale particles have received much attention as potentially useful materials exhibiting novel electronic, optical, magnetic, and biophotonic properties derived from the "quantum sized effect". In metal nanoparticles (NPs), the intraband contribution is modified through confinement of electrons within the NP. ${ }^{1}$ The localized surface plasmon (SP) is excited by an external electric field at a certain incident light frequency, resulting in an intense surface plasmon resonance (SPR) band. The resonance frequency and intensity of the SPR band is dependent on the kind of metal and is highly sensitive to the size, shape, size distribution, and surrounding medium of the metal NPs. ${ }^{2,3}$ Many researchers have studied the effects of the particle-size distributions and shapes on the behavior of NPs. ${ }^{1-4}$ Controlling the shape and size of NPs allows control over the wavelength of SPR resonance. ${ }^{5,6}$ Another way to obtain metal particles with desired SPR wavelengths is to fabricate them with asymmetric shapes. Lamarre et al. reported that the shapes of metal NPs change from spherical to prolate spheroids under heavy ion beam bombardment. ${ }^{7}$ Asymmetric metal particles can also be produced using complicated electrochemical reduction through modified synthesis methods, such as the two-step reduction of $\mathrm{Au}^{3+}$ onto preexisting gold nuclei ("seeds"). ${ }^{8-10}$ Using these approaches, the SPR wavelengths of the resulting asymmetric metal NPs cannot be further tuned under the influence of an external field.

Metal NPs embedded in a transparent dielectric matrix have received considerable attention as promising candidates for future all-optical photonic devices because of their attractive nonlinear optical properties. ${ }^{11}$ Ferroelectric materials have large dielectric constants and refractive indices, which can strongly influence the local fields experienced around metal NPs. ${ }^{12}$ The unique phenomena exhibited by ferroelectric materials such as lead zirconate titanate (PZT) allow the fabrication of high-quality polycrystalline films exhibiting enhanced electric and ferroelec-

\footnotetext{
*E-mail: hsuenlichen@ntu.edu.tw.

${ }^{\dagger}$ Department of Materials Science and Engineering, National Taiwan University.

¥ National Nano Device Laboratory.
}

tric properties relative to those of bulk ceramics. Furthermore, PZT films can provide a sufficient change in refractive index and thickness when an electric field is applied, making them suitable for application in electro-optical and piezoelectric devices. ${ }^{13-15}$ PZT films incorporating metal NPs have been prepared using a complicated aerosol deposition method (ADM). ${ }^{16,17}$ In this method, individual particles of the depositing material are mixed with a carrier gas in the aerosol chamber to form an aerosol flow, which is ejected through a micro-orifice nozzle and deposited onto the substrate in a vacuum chamber.

Recently, Mulvaney demonstrated that the self-assembly of optically active gold nanoparticles with varying generations of poly (amidoamine) (PAMAM) dendrimers provides aggregates with controlled interparticle spacing for shifting the SPR wavelength. ${ }^{18}$ Nevertheless, using an external electric field to modulate SPR phenomena in nanocomposite materials in a cyclic manner has not been reported yet. In this paper, we demonstrate the use of a conventional organic chemical addition method to readily prepare Au NP-embedded PZT composite films. Combining the ferroelectric properties of PZT with the SPR phenomena of Au NPs, we studied the tunability of the optical properties of the nanocomposite metal-dielectric systems under applied electric fields. The shape-dependence of the optical properties in metal NPs can be used to extract the structure of a nanocomposite material. For example, optical spectra can be simulated with the Mie theory by fitting the measured dielectric function of the metal particles and their surrounding media. ${ }^{19,20}$ When an electric field was applied to the nanocomposite films, the refractive index of the surrounding PZT films and the shape of the metal NPs were both changed as a result of the piezoelectric properties of PZT. Combining Mie theory simulation with spectroscopic ellipsometer measurement, we estimated the degrees of deformation of the metal NPs and the changing refractive indices of the host PZT matrix under various applied electric fields.

\section{Experimental Section}

An organic chemical addition and phase-transfer method was used to prepare $\mathrm{Au} / \mathrm{PZT}$ nanocomposite films. $\mathrm{HAuCl}_{4} \cdot 3 \mathrm{H}_{2} \mathrm{O}$ from Aldrich, dodecylamine (98\%), toluene (99.5\%), and 


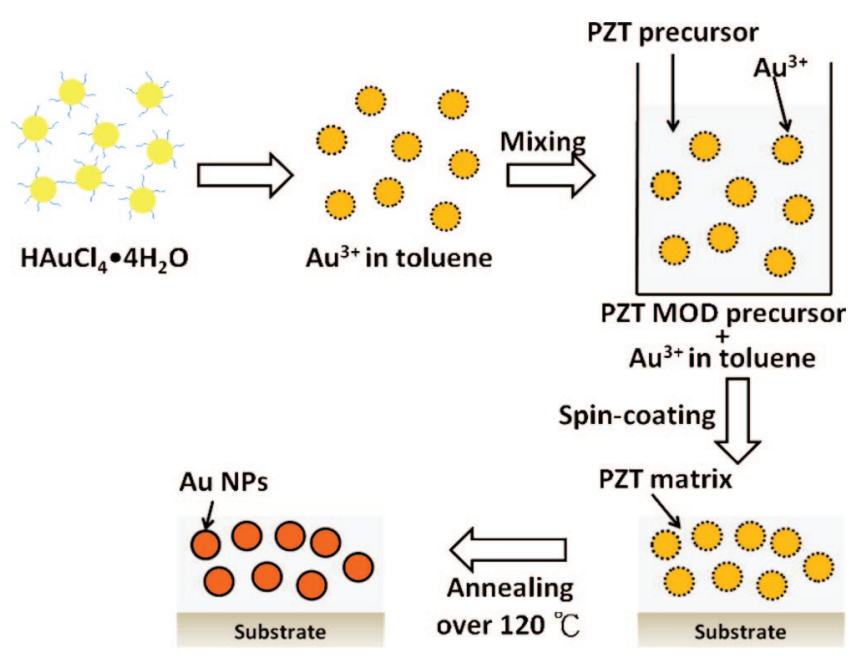

Figure 1. Schematic of organic chemical addition method of nanocomposite films.

ethanol (99\%) from ACROS were used as received. Deionized water was used for preparing aqueous solutions. A solution of dodecylamine-stabilized chloroaurate ions $\left(\mathrm{AuCl}_{4}{ }^{-}\right)$in toluene was prepared to allow the formation of a high concentration of $\mathrm{Au}$ NPs. The solutions of the PZT precursor and the $\mathrm{AuCl}_{4}{ }^{-}$ ions were prepared separately, and mixed together to form the composite precursor solution. The $\mathrm{Pb}\left(\mathrm{Zr}_{0.52} \mathrm{Ti}_{0.48}\right) \mathrm{O}_{3}$ (10 wt \%) precursor was prepared using the metal-organic decomposition (MOD) method (Kojundo, Japan).

The solution of dodecylamine-stabilized $\mathrm{AuCl}_{4}{ }^{-}$ions in toluene was prepared using a modified procedure, as shown in Figure $1 .^{9,21}$ A $50 \mathrm{mM}$ aqueous $\mathrm{HAuCl}_{4}$ solution $(5 \mathrm{~mL})$ was mixed with an ethanol solution $(5 \mathrm{~mL})$ containing dodecylamine $(1 \mathrm{~mL})$. After the mixture was stirred for $1 \mathrm{~min}$, toluene $(5 \mathrm{~mL})$ was added, and then the mixture was stirred for an additional 3 min. Phase transfer of the dodecylamine-stabilized $\mathrm{AuCl}_{4}{ }^{-}$ions from water to toluene occurred quickly and completely, as evidenced by the complete bleaching of the color from the aqueous phase. The concentration of $\mathrm{AuCl}_{4}{ }^{-}$ions in the toluene-assuming complete transfer from the aqueous phasewas, therefore, $50 \mathrm{mM}$. The hydrophobicized $\mathrm{AuCl}_{4}{ }^{-}$ions in toluene were separated from the aqueous phase and stored for further experiments.

A solution of $\mathrm{AuCl}_{4}{ }^{-}$ions was added into the MOD precursor of PZT; Au NPs formed during high-temperature heating process as a result of the decomposition of the $\mathrm{AuCl}_{4}{ }^{-}$-dodecylamine complexes. Au/PZT nanocomposite films were fabricated using the following procedure. The PZT solution (1 g) was mixed with $25 \mathrm{mM}$ organic-phase $\mathrm{HAuCl}_{4}(0.1,0.5$, or $1 \mathrm{~mL})$. The precursors were uniformly stirred for $5 \mathrm{~min}$ and then coated on a glass or $\mathrm{SrTiO}_{3}$ (STO) substrate using a spin-coater. The nanocomposite films were formed and dried at $120{ }^{\circ} \mathrm{C}$ for 30 min and then heated at $450{ }^{\circ} \mathrm{C}$ for $30 \mathrm{~min}$ in air for pyrolysis. Finally, some of the samples were sintered at $650^{\circ} \mathrm{C}$ for $1 \mathrm{~h}$ in an oven to form a more complete perovskite phase. The ferroelectric nanocomposite films were fabricated on a $\mathrm{SrTiO}_{3}$ bottom electrode and covered with indium tin oxide (ITO) glass as the top electrode to form metal-insulator-metal structures that could be subjected to an applied external electric field.

The crystalline structures of the Au/PZT films were analyzed using an X-ray diffractometer (XRD) with $\mathrm{Cu} \mathrm{K} \alpha$ radiation (Rigaku); the microstructure of composite films was analyzed using a high-resolution transmission electron microscope (HRTEM; JEM 2010, JEOL). The TEM samples were prepared

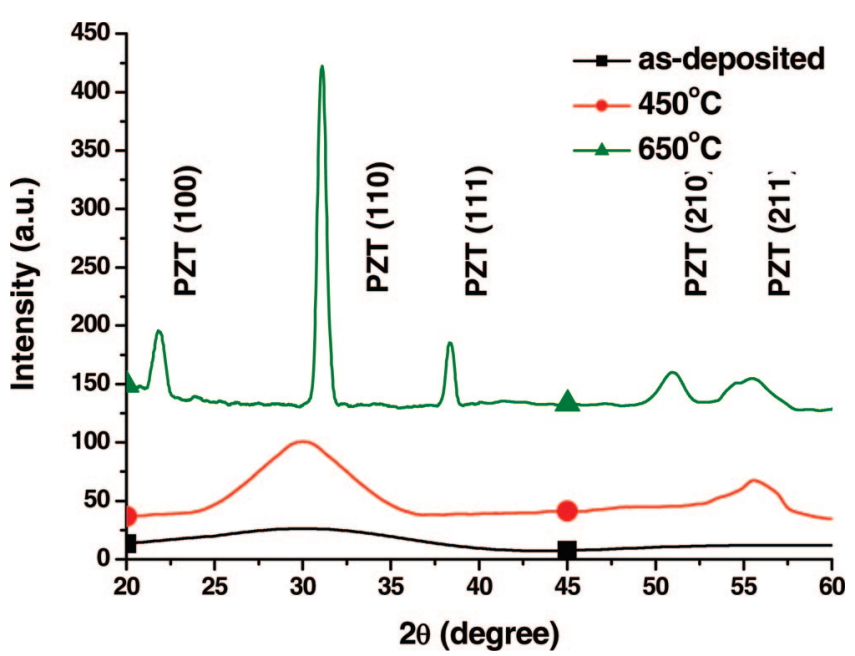

Figure 2. XRD spectra of the Au/PZT nanocomposite films prepared using various annealing temperatures.

using a conventional polish and ion milling method. The optical spectra were measured using a spectrophotometer (U4100, Hitachi). The ellipsometric parameters $\Psi$ and $\Delta$ of the PZT films were measured using an ellipsometer (SE5, Sopra). The ellipsometric data were fitted using a classical Lorentz oscillator model for the refractive indices.

\section{Results and Discussion}

Figure 2 displays X-ray diffraction spectra of Au/PZT composite films prepared using $1 \mathrm{~mL}$ of the precursor $\mathrm{HAuCl}_{4}$ solution. When the annealing temperature was larger than $450{ }^{\circ} \mathrm{C}$, the composite films crystallized. The (100), (111), and (210) planes of PZT did not appear in the X-ray diffraction spectrum of the sample prepared at $450{ }^{\circ} \mathrm{C}$, corresponding to a pyrochloric phase. The crystalline size was determined by considering the full width at half-maximum (fwhm) of the signals in the X-ray diffraction spectra. Further annealing at $650{ }^{\circ} \mathrm{C}$ provided composite films that were more crystalline and dense, as evidenced by the smaller values of fwhm of the (110), (100), (111), (210), and (211) bands. The crystalline size was ca. 3 and $37 \mathrm{~nm}$ at 450 and $650{ }^{\circ} \mathrm{C}$, respectively, suggesting that these crystalline composite films should possess improved ferroelectric properties.

Figure 3 presents TEM images and the energy dispersive spectrometric (EDS) analysis of the Au/PZT composite films prepared using $1 \mathrm{~mL}$ of the $\mathrm{HAuCl}_{4}$ precursor solution and annealing at various temperatures. Figure 3 a reveals the absence of $\mathrm{Au}$ NPs in the PZT matrix, which we confirmed through EDS analysis. The organic solvent was removed from the nanocomposite films at $120{ }^{\circ} \mathrm{C}$, but the perovskite structure of the nanocomposite films did not form at this low temperature. The TEM image in Figure $3 \mathrm{~b}$ displays Au NPs embedded in the PZT matrix annealed at $450{ }^{\circ} \mathrm{C}$. The TEM and EDS analyses confirmed the composition of Au NPs and the polycrystalline structure of the nanocomposites. Similarly, when the Au/PZT composite films were annealed at $650{ }^{\circ} \mathrm{C}$, they become denser, with the perovskite structure becoming more apparent. This higher annealing temperature improved the ferroelectric properties of the Au/PZT composite films as a result of the increased crystal size (Figure 3c) and recovery of lattice distortion. These results suggest that the Au NPs were dispersed homogeneously in the PZT matrix and that the particle-size increased upon increasing the annealing temperature.

The optical properties of metal particles embedded in a dielectric matrix can be described using the Mie theory. ${ }^{19,20}$ 

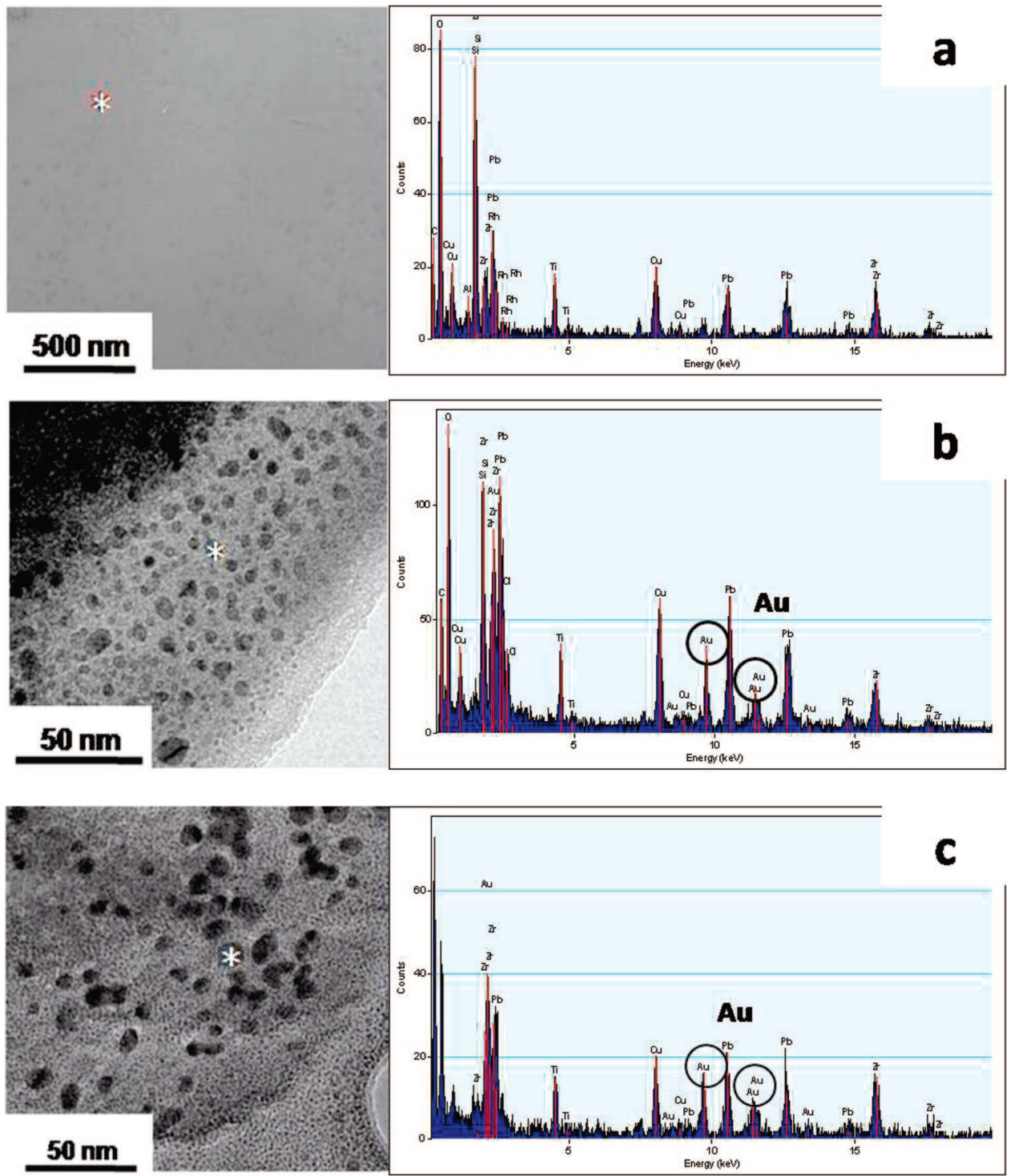

Figure 3. TEM images and EDS analyses of the Au/PZT nanocomposite films prepared using annealing temperatures of (a) 120, (b) 450, and (c) $650{ }^{\circ} \mathrm{C}$.

Well-separated metallic NPs having dimensions significantly smaller than the wavelength of the exciting light are characterized by a broad, intense SPR absorption band. The bandwidth, peak height, and position of the absorption maximum depend strongly on the size, size distribution, spatial distribution, and shape of the metallic NPs, as well as on the nanostructure and refractive index of the host matrix. When the metal NPs are embedded in the matrix of an electro-optical (EO) material, such as a PZT film, the SPR of the nanocomposite film can be modulated by the EO effect of the matrix. At a suitable annealing temperature, perovskite structures in PZT films can be formed to generate an additional electric field, $E_{\text {polar }}$, outside of the Au NPs as a result of a self-polarization effect. The refractive index of the PZT film would, therefore, change upon applying an external electric field. ${ }^{22}$

Figure 4 displays optical transmission spectra of nanocomposite films prepared using various $\mathrm{HAuCl}_{4}$ contents and annealing temperatures. For the nanocomposite films annealed at 120,450 , and $650{ }^{\circ} \mathrm{C}$, the SPR absorption bands were located at ca. 550, 580, and $615 \mathrm{~nm}$, respectively, consistent with an increase in the particle size of the Au NPs in the nanocomposite films; i.e., larger Au NPs exhibit red-shifted SPR absorption bands. ${ }^{5}$ The TEM images in Figure 3, parts b and c, confirm that the Au NPs increased in size upon increasing the annealing temperature.

Figure 5 displays ferroelectric hysterics curves of the PZT and Au/PZT nanocomposite films after annealing at $650{ }^{\circ} \mathrm{C}$. Both films exhibit apparent piezoelectric properties. The $\mathrm{Au} /$ PZT nanocomposite films featured smaller remanent polarizations and coercive electric fields, which we suggest arise from the $\mathrm{Au}$ NPs not only preventing some portions of PZT from forming perovskite structures but also influencing the distribution of the electric field. The presence of the Au NPs prevented shrinkage of the PZT films during the thermal annealing process and affected the density and structure of the PZT matrix. Nevertheless, the apparent piezoelectric and EO properties of the Au/PZT nanocomposite films were sufficiently high for them to be used in the active modulation of SPR wavelengths under an applied electric field.

Figure 6 displays the SPR absorption spectra of nanocomposite films with the thickness ca. $400 \mathrm{~nm}$ prepared using 1 $\mathrm{mL}$ of the $\mathrm{HAuCl}_{4}$ precursor solution and annealing at $650{ }^{\circ} \mathrm{C}$ under various external potentials. Without the application of an external electric field, the SPR absorption peak appeared at 


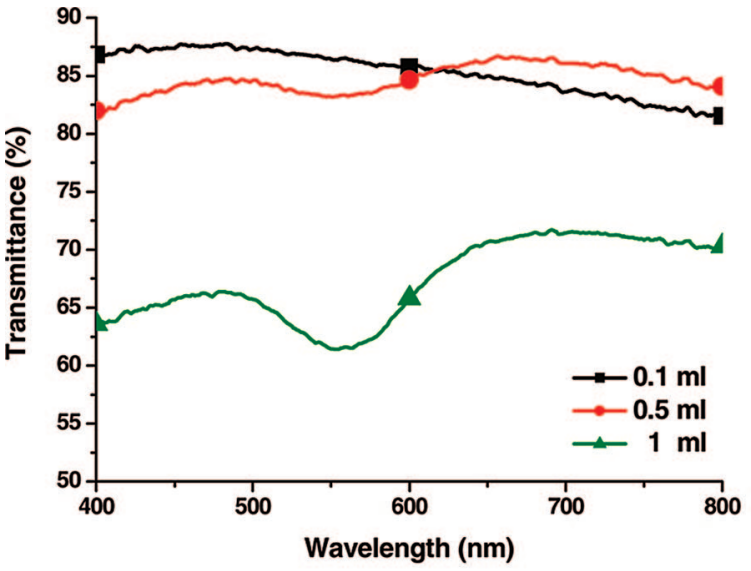

(a)

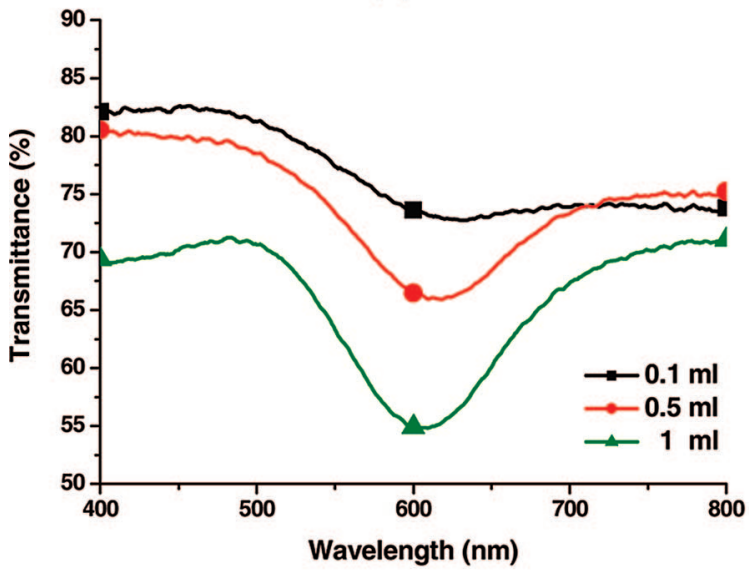

(c)

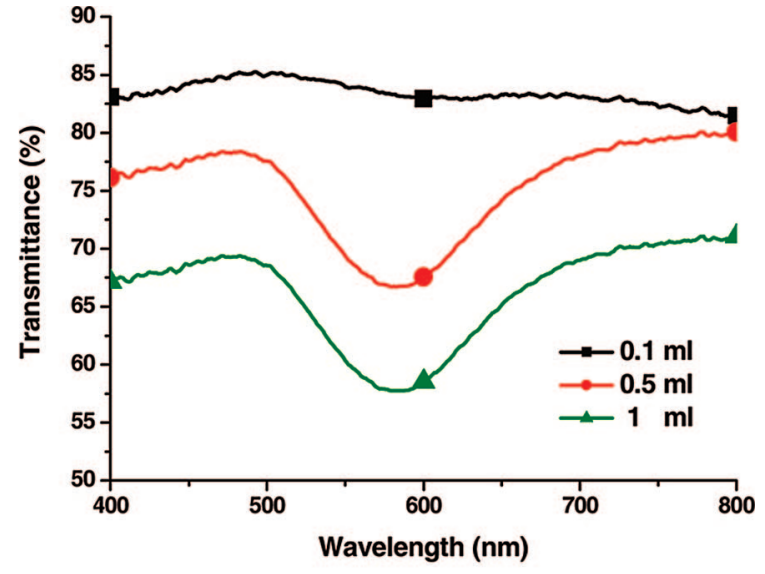

(b)

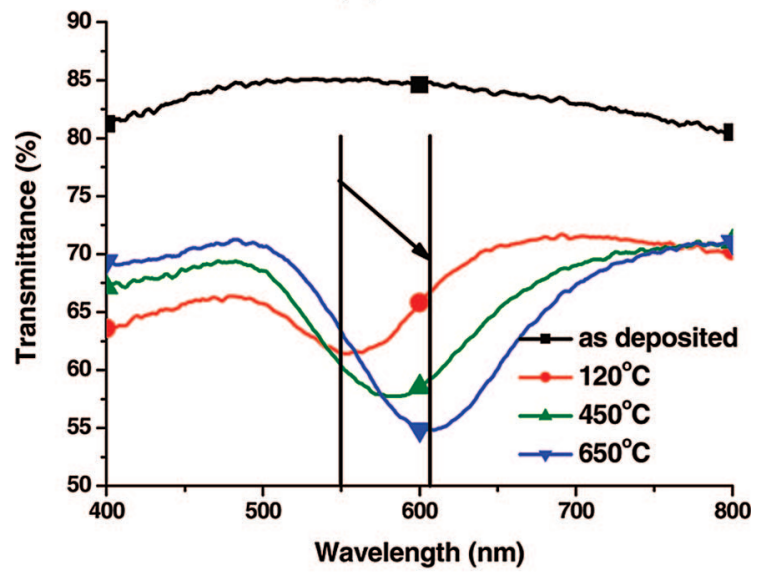

(d)

Figure 4. Transmittance spectra of the nanocomposite films prepared using various $\mathrm{HAuCl}_{4}$ contents and annealing temperatures of (a) 120 , (b) 450 , and (c) $650{ }^{\circ} \mathrm{C}$. (d) Transmittance spectra of the nanocomposite films prepared using $1 \mathrm{~mL}$ of the $\mathrm{HAuCl}_{4}$ precursor solution.

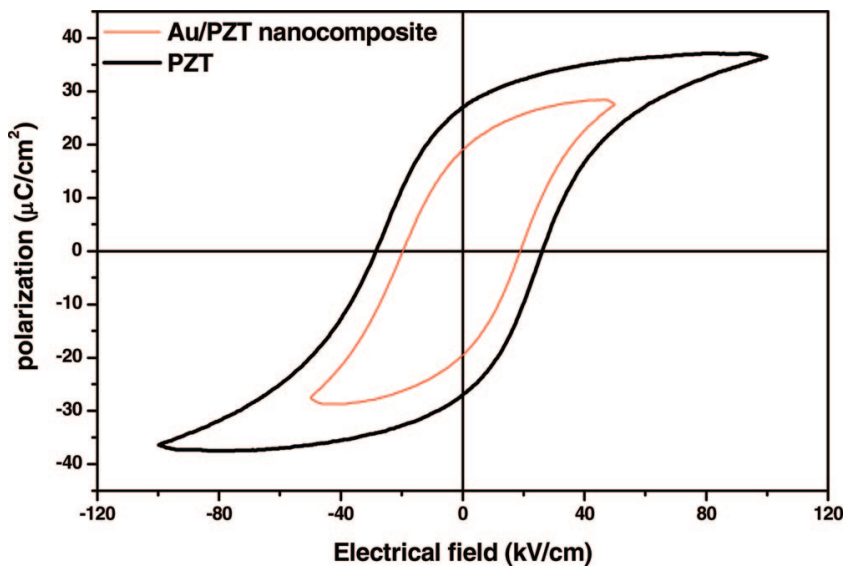

Figure 5. Ferroelectric hysteresis curves of the PZT and Au/PZT nanocomposite films after annealing at $650{ }^{\circ} \mathrm{C}$.

$615 \mathrm{~nm}$; when electric fields of 50,250 , and $500 \mathrm{kV} / \mathrm{cm}$ were applied, the SPR signals red-shifted to 618,625 , and $649 \mathrm{~nm}$, respectively. When we removed the external voltage, after several days the SPR absorption signal returned to its initial wavelength of $615 \mathrm{~nm}$. Thus, we were able to cycle the modulation of the SPR wavelength through the application of an electric field to $\mathrm{Au}$ NPs embedded within nanocomposite films.

The size-distribution of Au NPs is a factor affecting the width of its SPR absorption band. The TEM image in Figure 3c

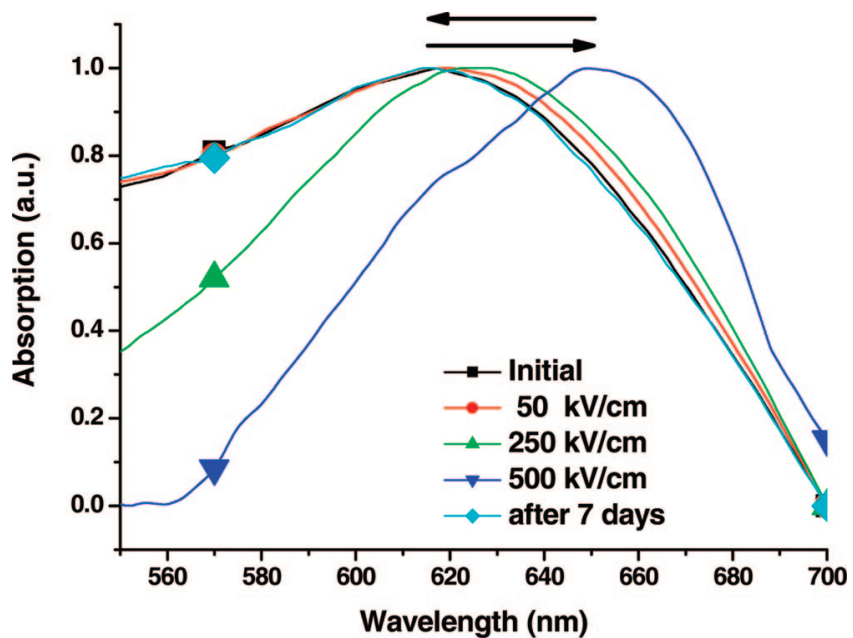

Figure 6. Transmission spectra of the nanocomposite films subjected to various external electric fields.

suggests that the sizes of the Au NPs in the PZT matrix were ca. 2-12 nm, with an average diameter of ca. $6.4 \mathrm{~nm}$ from the image. As indicated in Figure 5, the large distribution of the Au NPs' sizes in the nanocomposite film increased the bandwidth of the SPR absorption.

To study the modulation of the SPR absorption band in the nanocomposite films, we used the Mie theory to simulate the changing SPR absorption under an applied electric field. In 
TABLE 1: Measured and Simulated SPR Absorption Signals at Various External Electric Fields, Incorporating the Measured Refractive Indices of the Surrounding PZT

\begin{tabular}{lllll}
\hline applied electric field $(\mathrm{kV} / \mathrm{cm})$ & initial & 50 & 250 & 500 \\
measured SPR peaks $(\mathrm{nm})$ & 615 & 618 & 625 & 649 \\
$\begin{array}{c}\text { Measured refractive index of } \\
\quad 2.376\end{array}$ & 2.378 & 2.390 & 2.500 \\
$\begin{array}{c}\text { surrounding PZT } \\
\text { simulated SPR peaks (nm) }\end{array}$ & 616 & 618 & 623 & 645
\end{tabular}

the Mie theory, many factors will shift the SPR absorption band to a longer wavelength, including a larger particle-size, a larger refractive index of the surrounding medium, and asymmetry in the structures of the metal NPs. In this study, we used an ellipsometer to measure the refractive index change of the PZT films after applying the electric field. Table 1 lists the measured refractive indices of the PZT films after applying external electric fields of 50,250 , and $500 \mathrm{kV} / \mathrm{cm}$. An increase of the refractive index of the PZT films would shift the SPR absorption band of the nanocomposite films to longer wavelength.

In using the Mie theory, we assumed that the Au particle size, shape, distribution, and optical properties did not change during the application of the electric field. After incorporating the measured refractive indices into the calculations, Figure 7 indicates that the simulated SPR absorption signals were redshifted upon increasing the measured refractive index of the surrounding medium. Before applying an electric field, the measured refractive index of the surrounding PZT was 2.376; the simulated SPR peak appeared at $616 \mathrm{~nm}$ for Au NPs having a diameter of $2.3 \mathrm{~nm}$ for matching both the measured SPR peak and measured refractive index of PZT films. After applying external electric fields of 50,250 , and $500 \mathrm{kV} / \mathrm{cm}$, the measured refractive indices of PZT were 2.378, 2.390, and 2.500, respectively; the simulated SPR absorption peaks are 618,623 , and $645 \mathrm{~nm}$, respectively. In this study, the relative changes of refractive index in nanocomposite films were $0.002,0.014$, and 0.124 under the electric fields of 50,250 , and $500 \mathrm{kV} / \mathrm{cm}$, respectively. These values were smaller than those reported in the literatures. ${ }^{22}$ This was because the Au NPs in the PZT matrix prevented some portions of PZT from forming perovskite structures, and also influenced the distribution of electric field in nanocomposite films.

The experimental and calculated results presented in Table 1 matched well when the applied electric fields were 0 and 50 $\mathrm{kV} / \mathrm{cm}$; in contrast, the difference between measured and simulated SPR absorption peaks were more apparent with the

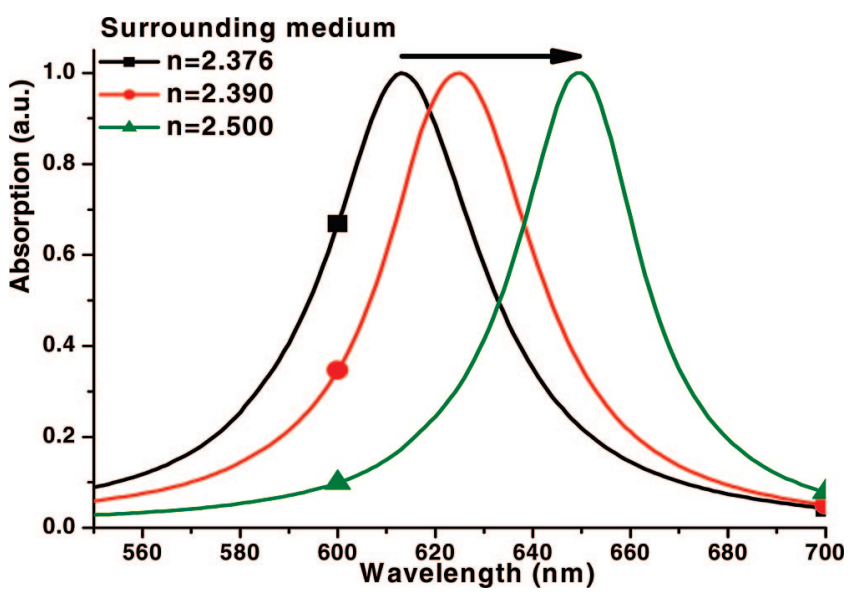

Figure 7. Simulated spectra of the Au/PZT nanocomposite films under various applied external electric fields, using the measured refractive indices of the PZT films.
TABLE 2: Measured and Simulated Refractive Indices of PZT Media at Various External Electric Fields, Incorporating the SPR Absorption Signals

\begin{tabular}{lllll}
\hline applied electric field $(\mathrm{kV} / \mathrm{cm})$ & initial & 50 & 250 & 500 \\
measured SPR peaks $(\mathrm{nm})$ & 615 & 618 & 625 & 649 \\
$\begin{array}{c}\text { Measured refractive indices of } \\
\text { uniform PZT films }\end{array}$ & 2.376 & 2.378 & 2.390 & 2.500 \\
$\begin{array}{c}\text { simulated refractive indices of } \\
\quad \text { surrounding PZT medium }\end{array}$ & 2.263 & 2.292 & 2.312 & 2.478 \\
& & & &
\end{tabular}

applied electric fields were 250 and $500 \mathrm{kV} / \mathrm{cm}$ (ca. 2 and 4 $\mathrm{nm}$, respectively), possibly because of deformation of the shapes of the Au NPs. When applying an electric filed, not only does the refractive index change- so too does the volume of the Au/ PZT nanocomposite film. We suggest that the embedded Au NPs were deformed as a result of the changing volume of the PZT matrix. When applying a large external electric field, the piezoelectric properties of the PZT films induce the elongation of the PZT, which would compress the spherical Au NPs into ellipsoids. In the simulation above, we assumed that the $\mathrm{Au}$ NPs were spheres, i.e., their aspect ratio was equal to one. Upon increasing the aspect ratio of elliptic Au NPs, the simulated SPR peaks became red-shifted. Therefore, we could estimate the extent of deformation of the Au NPs by comparing the experimental values with those simulated using the Mie theory. We estimated that the deformation of Au NPs was ca. 3.4\% under an applied external electric field of $500 \mathrm{kV} / \mathrm{cm}$.

From the TEM image in Figure 3c, the average diameter of $\mathrm{Au}$ NPs was ca. $6.4 \mathrm{~nm}$. By using the particle-size of $6.4 \mathrm{~nm}$, the simulated SPR peaks were larger than the measured peaks. Consequently, we propose another assumption for matching the measured SPR peaks and particle-size in the TEM image. Refractive indices of PZT medium around gold NPs in the nanocomposite films are different to the refractive indices of uniform PZT films. For matching the measured SPR peaks and particle-size in the TEM image, Table 2 displays the measured refractive indices of uniform PZT films and simulated refractive index of surrounding PZT medium under different external electric fields. We find that simulated refractive indices of surrounding PZT medium are slightly smaller than the measured refractive indices of uniform PZT films. The smaller refractive index of surrounding PZT medium may be due to the increased void or crack in nanocomposite matrix as Au NPs embedded into PZT films.

Therefore, the major factor influencing the shift in the SPR absorption wavelength was the change in refractive index of the host matrix in the Au/PZT nanocomposite films under applied electric fields. Thus, we could readily adjust the position of the SPR absorption band of Au/PZT nanocomposite films by varying the strength of the applied electric field. Therefore, modulation of SPR phenomena was accomplished by altering the piezoelectric properties of Au/PZT nanocomposite films. This composite system has great potential for use in biophotonics, tunable optical filters, and optical switching devices.

\section{Conclusions}

In this paper, we demonstrate a simple method for preparing gold/lead zirconate titanate nanocomposite films using organic chemical addition and phase-transfer methods. Applying an external electric field after the composite films had been prepared allowed their SPR phenomena to be modulated. From the analysis of TEM images and XRD data, it appeared that the Au NPs were embedded homogeneously in the PZT matrix and that the sizes of the PZT crystals and metal particles both 
increased upon increasing the annealing temperature. Higher annealing temperatures improved the ferroelectric properties of the $\mathrm{Au} / \mathrm{PZT}$ composite films as a result of increasing the crystal size and recovering lattice distortion. The SPR spectra of nanocomposite films prepared using various $\mathrm{HAuCl}_{4}$ contents and annealing temperatures revealed that the intensity of the SPR absorption band increased upon increasing the $\mathrm{HAuCl}_{4}$ content and that larger Au NPs led to a red-shifting of the SPR absorption band upon increasing the annealing temperature. We also observed that the films of both PZT and the Au/PZT nanocomposites exhibited apparent piezoelectric properties in their ferroelectric hysterics curves. The apparent piezoelectric and EO properties of the Au/PZT nanocomposite films could be used for active modulation of the SPR wavelengths. By applying an electric field to the Au NP-embedded nanocomposite films, cyclic modulation of the SPR wavelength was achieved. The major factor influencing the shift of the SPR absorption was the refractive index change of the host PZT matrix in the $\mathrm{Au} / \mathrm{PZT}$ nanocomposite films when an electric field was applied.

Acknowledgment. The authors acknowledge the support from the National Science Council, Taiwan, R.O.C., under Project NSC-96-2623-7-002-005-ET.

\section{References and Notes}

(1) Hutter, E.; Fendler, J. H. Adv. Mater. 2004, 16, 1685.

(2) Underwood, S.; Mulvaney, P. Langmuir 1994, 10, 3427.
(3) Mulvaney, P. Langmuir 1996, 12, 788.

(4) Dalacu, D.; Martinu, L. J. Opt. Soc. Am. B 2001, 18, 85.

(5) Kelly, K. L.; Coronado, E.; Zhao, L. L.; Schatz, G. C. J. Phys. Chem. B 2003, 107, 668.

(6) Sosa, I. O.; Noguez, C.; Barrera, R. G. J. Phys. Chem. B 2003, 107,6269 .

(7) Lamarrea, J. M.; Yub, Z.; Harkatib, C.; Roordab, S.; Martinua, L. Thin Solid Films 2005, 479, 232.

(8) Nikoobakht, B.; El-Sayed, M. A. Chem. Mater. 2003, 15, 1957.

(9) Jana, N. R.; Gearheart, L. A.; Murphy, C. J. J. Phys. Chem. B 2001, 105,4065 .

(10) Perez-Juste, J.; Liz-Marzán, L. M.; Carnie, S.; Chan, D. Y. C.; Mulvaney, P. Adv. Funct. Mater. 2004, 14, 571.

(11) Chakraborty, P. J. Mater. Sci. 1998, 33, 2235.

(12) Zhou, J.; Li, L.; Gui, Z.; Zhang, X. Ferroelectrics 1997, 196, 85.

(13) Huang, Z.; Meng, X.; Yang, P.; Zhang, Z.; Chu, J. Appl. Phys. Lett. 2000, 76, 3980.

(14) Moret, M. P.; Devillers, M. A. C.; Worhoff, K.; Larsen, P. K. J. Appl. Phys. 2002, 92, 468.

(15) Yang, S.; Mo, D.; Tang, X. Ferroelectrics 2003, 287, 35.

(16) Dalacu, D.; Martinu, L. Appl. Phys. Lett. 2000, 77, 4283.

(17) Dalacu, D.; Martinu, L. J. Appl. Phys. 2000, 87, 228.

(18) Srivastava, S.; Frankamp, B. L.; Rotello, V. M. Chem. Mater. 2005, 17, 487.

(19) Mie, G. Ann. Phys. (Leipzig) 1908, 25, 377.

(20) Kelly, K. L.; Coronado, E.; Zhao, L. L.; Schatz, G. C. J. Phys. Chem. B 2003, 107, 668.

(21) Yang, J.; Lee, J. Y.; Too, H. P. J. Phys. Chem. B 2005, 109, 19208.

(22) Potter, Jr.; B, G.; Sinclair, M. B.; Dimos, D.; Tuttle, B. A.; Schwartz, R. W. J. Non-Cryst. Solids 1994, 178, 69.

JP711828K 